\title{
Therapeutic potential of living donor liver transplantation from heterozygous carrier donors in children with propionic acidemia
}

\author{
Zhi-Gui Zeng ${ }^{1,3 \dagger}$, Guang-Peng Zhou ${ }^{1,3 \dagger}$, Lin Wei ${ }^{1,3}$, Wei Qu ${ }^{1,3}$, Ying Liu ${ }^{1,2,3}$, Yu-Le Tan ${ }^{1,3}$, Jun Wang ${ }^{1,3}$, \\ Li-Ying Sun ${ }^{1,2,3^{*}}$ and Zhi-Jun Zhu ${ }^{1,3^{*}}$ (1)
}

\begin{abstract}
Background: Current world experience regarding living donor liver transplantation (LDLT) in the treatment of propionic acidemia (PA) is limited, especially in terms of using obligate heterozygous carriers as donors. This study aimed to evaluate the clinical outcomes of LDLT in children with PA.

Methods: From November 2017 to January 2020, 7 of the 192 children who underwent LDLT at our institution had been diagnosed with PA (median age, 2.1 years; range, 1.1-5.8 years). The primary indication for transplantation was frequent metabolic decompensations in 6 patients and preventative treatment in 1 patient. Of the seven parental living donors, six were genetically proven obligate heterozygous carriers.

Results: During a median follow-up of 23.9 months (range, 13.9-40.2 months), all patients were alive with 100\% allograft survival, and no severe transplant-related complications occurred. In the case of liberalized protein intake, they did not suffer metabolic decompensation or disease-related complications and made progress in neurodevelopmental delay and body growth, as well as blood and urinary metabolite levels. In one patient with pre-existing mild dilated cardiomyopathy, her echocardiogram results completely normalized 13.8 months post-transplant. All living donors recovered well after surgery, with no metabolic decompensations or procedure-related complications. Western blotting revealed that the hepatic expressions of PCCA and PCCB in one of the heterozygous donors were comparable to those of the normal healthy control at the protein level.
\end{abstract}

Conclusions: LDLT using partial liver grafts from asymptomatic obligate heterozygous carrier donors is a viable therapeutic option for selected PA patients, with no negative impact on donors' and recipients' clinical courses.

Keywords: Propionic acidemia, Living donor liver transplantation, Heterozygous carrier, Propionyl-CoA carboxylase

\footnotetext{
*Correspondence: sunxlx@outlook.com; zhu-zhijun@outlook.com ${ }^{\dagger}$ Zhi-Gui Zeng and Guang-Peng Zhou contributed equally to this work. ${ }^{1}$ Liver Transplantation Center, National Clinical Research Center for Digestive Diseases, Beijing Friendship Hospital, Capital Medical University, No. 101 Lu Yuan Dong Road, Tong-Zhou District, Beijing 101100, China

Full list of author information is available at the end of the article
}

\section{Introduction}

Propionic acidemia (PA; Online Mendelian Inheritance in Man [OMIM] \#606054) is an ultrarare autosomal-recessive disorder of metabolism characterized by biallelic pathogenic variants on chromosome 13q32.3 $(P C C A)$ or 3q22.3 (PCCB), resulting in the deficiency of the mitochondrial enzyme propionyl-CoA carboxylase (PCC) [1]. Dysfunction of PCC fails to convert propionyl-CoA to methylmalonyl-CoA, thereby leading to the chronic accumulation of propionic acid and original author(s) and the source, provide a link to the Creative Commons licence, and indicate if changes were made. The images or other third party material in this article are included in the article's Creative Commons licence, unless indicated otherwise in a credit line to the material. If material is not included in the article's Creative Commons licence and your intended use is not permitted by statutory regulation or exceeds the permitted use, you will need to obtain permission directly from the copyright holder. To view a copy of this licence, visit http://creativecommons.org/licenses/by/4.0/. The Creative Commons Public Domain Dedication waiver (http://creativecommons.org/publicdomain/zero/1.0/) applies to the data made available in this article, unless otherwise stated in a credit line to the data. 
propionyl-CoA-related metabolites. These circulating toxic metabolites continuously cause damage to various organs and tissues throughout the body [2]. Since compound heterozygotes in most patients result in undefined genotype-phenotype correlation, affected individuals present with a broad spectrum of clinical manifestations, and age of onset ranges from the neonatal period to adulthood [3]. Characteristic patients usually present in infancy with poor feeding and episodic vomiting within the first few hours to days of life. Without prompt diagnosis and treatment, the illness can progress rapidly to severe ketoacidosis, hyperammonemia, and hyperlactatemia, manifesting as lethargy, seizure, or coma that can result in early death $[4,5]$. Despite good compliance with long-term conservative management consisting of individualized nutritional intervention, levocarnitine supplementation, and oral metronidazole, the overall prognosis of PA patients remains poor [6]. Patients surviving their initial metabolic decompensation episode may suffer frequent metabolic decompensations and disease-related long-term multiorgan sequelae such as growth impairment, neurocognitive deficits, cardiomyopathy, pancreatitis, or chronic kidney disease [7, 8]. Furthermore, PA patients' lifelong high-intensity medical and dietary management demands can severely affect family life and cause tremendous financial and psychosocial stress to patients' caregivers $[9,10]$.

Since replacing the enzyme-deficient liver with a metabolically normal liver could help regain partial PCC activity, after the first attempt in 1991, liver transplantation (LT) has emerged as a novel therapeutic option for selected PA patients who, despite strict dietary and medical intervention, still experience frequent metabolic decompensations or cardiomyopathy [11-15]. In some centers, early LT has been offered as a preventative treatment for metabolically stable pediatric PA patients without metabolic decompensation and severe neurological or cardiac sequelae $[13,14,16]$.

Living donors, especially in countries with limited availability of deceased donors, are an essential source of donor organs. And parents of liver transplant candidates are often the predominant and even sole source of living donors. Thus, living donor liver transplantation (LDLT) using an allograft from a heterozygous carrier donor in autosomal-recessive disorders is always inevitable. However, there remains a concern for the potentiality of the insufficient PCC activity in the donor remnant liver and the partial liver graft, and the current world experience of using obligate heterozygous carriers in LDLT for PA patients is limited. Here, we report our single-center experience with LDLT in 7 children with PA, of whom 6 received partial liver grafts from genetically proven heterozygous parental living donors.

\section{Materials and methods Data collection}

Patients with PA who underwent LT at our hospital between November 2017 and January 2020 were identified from the liver transplant recipient database. We retrospectively collected demographic data (gender, age, height, weight, age and features of initial clinical manifestations, age at diagnosis, daily protein intake, and medical therapy); transplantation details (indication for LT, age at LT, donor type, graft type, duration of intensive care unit and hospital stay, and postoperative LT-related complications); and laboratory measures (molecular genetic testing, urine organic acid, blood amino acid analysis, blood acylcarnitine profile, renal function, and echocardiography). The neurodevelopmental delay was assessed using the developmental quotient (DQ), which was calculated using the Griffiths Development ScalesChinese (GDS-C) and the Wechsler Intelligence Scale for Children-IV (WISC-IV) [17]. The hepatic expressions of PCCA and PCCB were assessed at the protein level by western blotting using antibodies directed against PCCA and PCCB proteins (anti-PCCA rabbit polyclonal antibody (ab154254), anti-PCCB rabbit polyclonal antibody (ab96729), Abcam).

\section{Statistical analysis}

Results were expressed as mean \pm standard deviation or median (range). Paired sample Student's $t$ test was used for comparative analysis of results before and after transplantation, and $P<0.05$ was considered a significant difference. Statistical analyses were performed using SPSS software 26.0 (SPSS, Inc., Chicago, IL, USA).

This study was reviewed and approved by the Ethical Committee of Hospital (No. 2020-P2-094-01) and conducted according to the ethical guidelines of the Declaration of Helsinki and the Declaration of Istanbul. We declare that all cases of LDLT were approved by the Ethical Committee of the Hospital, and all living donors were voluntary and altruistic.

\section{Results \\ Patient characteristics}

Between November 2017 and January 2020, 192 children underwent LDLT at our institution, of whom 7 (3.7\%) were diagnosed with PA. According to the age at initial presentation, 3 patients were early-onset (within the first month of life), and the remaining 4 were lateonset (after the first month). The diagnosis of PA was confirmed through a combination of clinical manifestations, typical biochemical findings, and identification of pathogenic variants in $P C C A$ or $P C C B$ on molecular genetic testing (Table 1; Fig. 1). No patients were from consanguineous parents or had a previously affected 


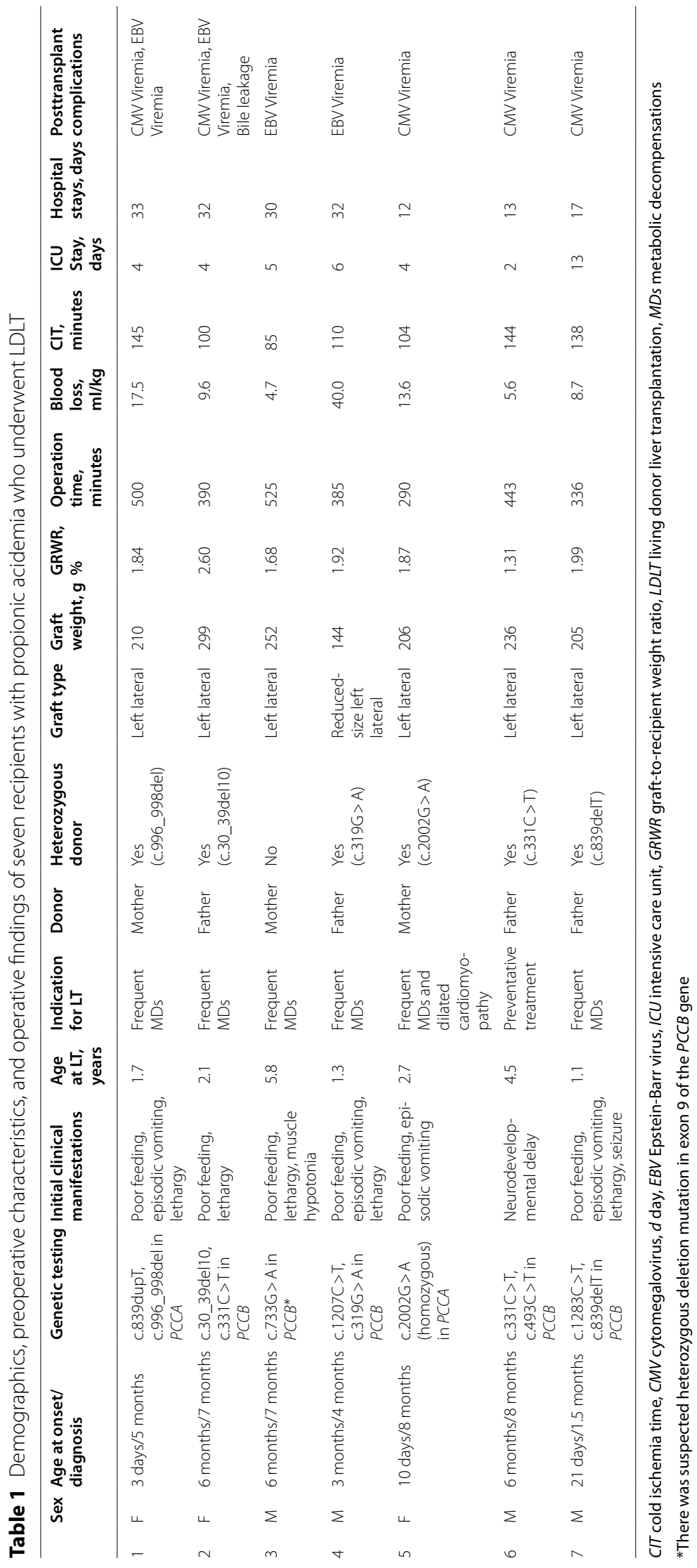




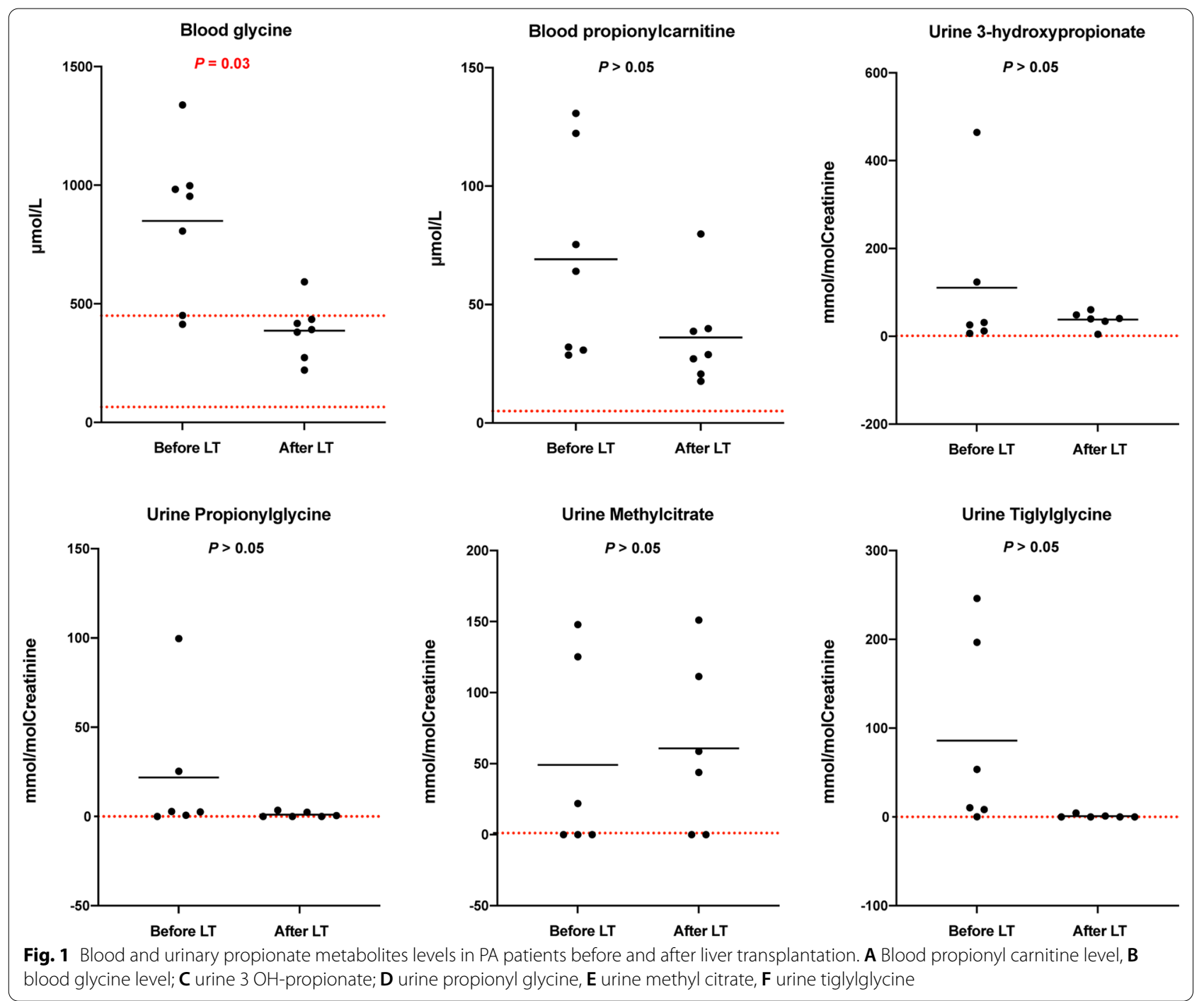

relative. After diagnosis, all patients followed a strict protein-restriction diet, a special formula that contains no isoleucine, methionine, threonine, and valine, and were administered levocarnitine supplementation. Before LT, the median protein restriction was $1.75 \mathrm{~g} / \mathrm{kg} /$ day (range, $0.8-2.7 \mathrm{~g} / \mathrm{kg} /$ day), and none required supportive feeding (Table 2).

\section{Living donor liver transplantation}

Patients underwent LDLT at a median age of 2.1 years (range, 1.1-5.8 years). The indications were frequent metabolic decompensations (6 patients) and preventative treatment (1 patient). All liver grafts were left lateral segments except for one reduced-size left lateral segment, voluntarily donated by their parents. Of the seven parental living donors, six were genetically proven obligate heterozygous carriers (Table 1). All recipients and donors underwent a smooth operation with stable intraoperative metabolic status. Immediate postoperative courses were uneventful, with no metabolic or hepatic decompensations episodes. The median intensive care unit and hospital stays were 4 days (range, 2-6 days) and 30 days (range, 12-33 days), respectively. Immunosuppressive therapy was based on tacrolimus and low-dose corticosteroids. Tacrolimus administration was started the day after LT, and the dose of tacrolimus was dynamically adjusted according to the target whole-blood trough level. The dose of steroids was gradually tapered and withdrawn within the first 6 months after transplantation. With a median follow-up of 23.9 months, all the recipients were alive with $100 \%$ allograft survival. Meanwhile, all living donors were discharged from the hospital, recovered well, and reported good working ability after surgery, with no procedure-related complications or metabolic acidosis during the perioperative period and throughout the follow-up period. 


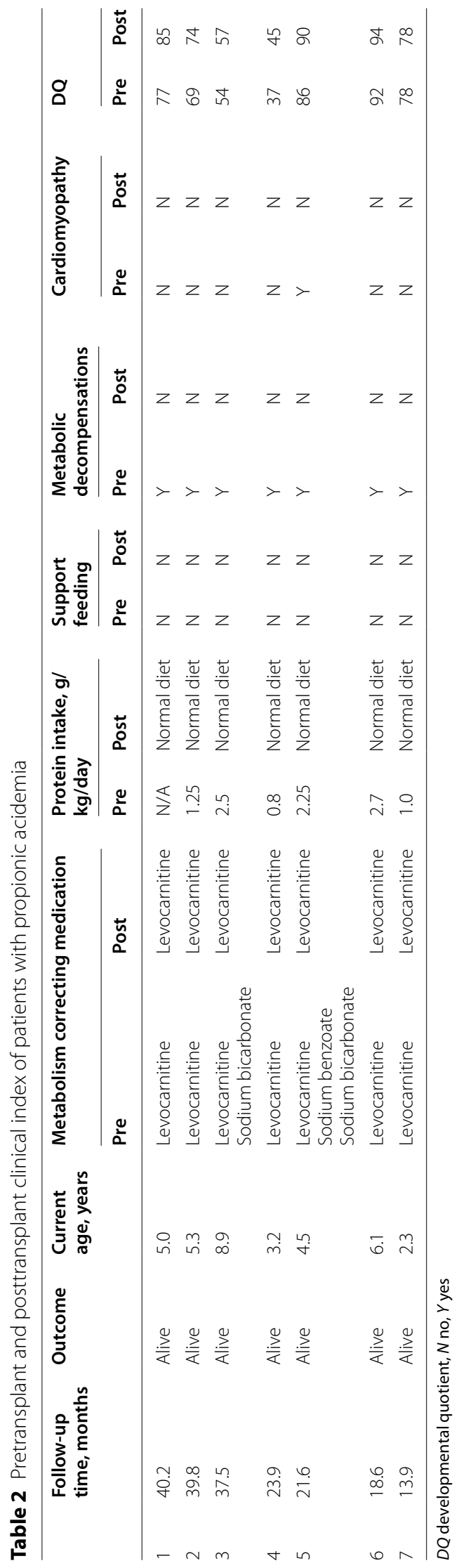


H\&E staining of the explanted diseased livers revealed normal liver lobules with the central vein, normal hepatic sinusoid, slightly loose and swollen hepatocytes, no hepatocyte necrosis or vacuolar degeneration, no fibrous tissue proliferation, with a few lymphocytes infiltrated in the partial portal area (Fig. 2A-G). The histopathological examination of the implanted donor livers indicated normal liver lobules with the central vein, normal hepatic sinusoid, and no hepatocyte necrosis or degeneration, with no or mild hepatocyte steatosis (Fig. $2 \mathrm{H}-\mathrm{N}$ ).

\section{Postoperative transplant-related complications}

No recipients developed any vascular complications after LT. A biliary leak was diagnosed in Patient 2, and she was managed conservatively. There was no occurrence of acute or chronic rejection in all 7 recipients during the follow-up period. Cytomegalovirus viremia and Epstein-Barr virus viremia were the most frequent infectious complications, which occurred in 5 and 4 patients, respectively. They all were treated with intravenous or oral ganciclovir. No patient developed suspected or confirmed posttransplant lymphoproliferative disease (PTLD).

\section{Postoperative metabolic consequences and diet}

There was no occurrence of metabolic decompensation in all 7 recipients during the follow-up period, allowing for progressive dietary protein liberalization without the need for any supplements other than levocarnitine. At the last follow-up, all patients had no formal protein restriction.

As part of the routine examination for patients with PA, propionate metabolites in blood [glycine and propionylcarnitine (C3)] and urine (3-hydroxypropionate, propionylglycine, methylcitrate, and tiglylglycine) were measured to evaluate metabolic improvement after LT. All seven patients, at least once, had blood glycine and
C3 levels recorded before and after LT. The mean blood glycine level was $849.17 \pm 326.59 \mu \mathrm{mol} / \mathrm{L}$ before $\mathrm{LT}$ and significantly decreased to $387.36 \pm 119.83 \mu \mathrm{mol} / \mathrm{L}$ after LT. This reduction of $461.81 \mu \mathrm{mol} / \mathrm{L}$ reached a statistical significance $(P=0.028)$, and the post-transplant mean glycine levels remained below the normal reference range $(65.0-450.0 \mu \mathrm{mol} / \mathrm{L})$. The mean values of blood C3 reduced from $69.12 \pm 43.14 \mu \mathrm{mol} / \mathrm{L}$ before $\mathrm{LT}$ to $36.10 \pm 21.00 \mu \mathrm{mol} / \mathrm{L}$ after LT. This reduction of $33.02 \mu \mathrm{mol} / \mathrm{L}$ did not reach a statistical significance $(P=0.17)$, and the post-transplant mean $C 3$ levels remained above the normal reference range (1.00$5.00 \mu \mathrm{mol} / \mathrm{L}$ ) (Fig. 1).

Six patients had, at least once, urine organic acid assay before and after LT. Compared with pretransplant values, urine 3-hydroxypropionate, propionylglycine, and tiglylglycine mean levels decreased to nearly normal levels after LT, while mean urine methylcitrate increased from $49.17 \pm 68.59 \mathrm{mmol} / \mathrm{mol}$ Creatinine before LT to $60.81 \pm 60.64 \mathrm{mmol} / \mathrm{mol}$ Creatinine after LT. However, this increase of $11.64 \mathrm{mmol} / \mathrm{mol}$ Creatinine did not reach a statistical significance $(P=0.61)$, and the posttransplant mean methylcitrate levels remained above the normal reference range $(0-1.00 \mathrm{mmol} / \mathrm{mol}$ Creatinine) (Fig. 1).

\section{Postoperative renal, cardiac, and neurological consequences}

Renal function was evaluated by serum creatinine and estimated glomerular filtration rate (eGFR) calculated according to simplified diet modification in renal disease equation. Values of serum creatinine and eGFR were both within the normal ranges in all patients before and after LT (Fig. 3).

As part of regular assessment, echocardiography was performed for all PA patients during the pretransplant evaluation and every 6 months to 1 year during the

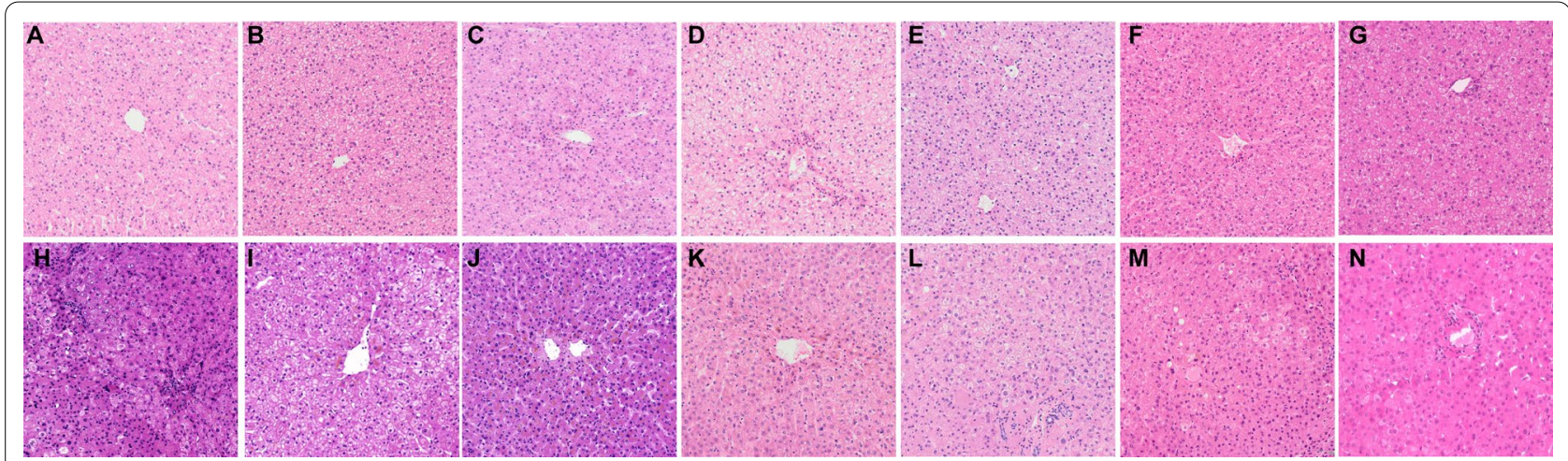

Fig. 2 The seven recipients' representative histological finding (H\&E staining, $100 \times)$ of the explanted diseased livers $(\mathbf{A}-\mathbf{G})$ and implanted donor livers $(\mathbf{H}-\mathbf{N})$ 
Serum creatinine

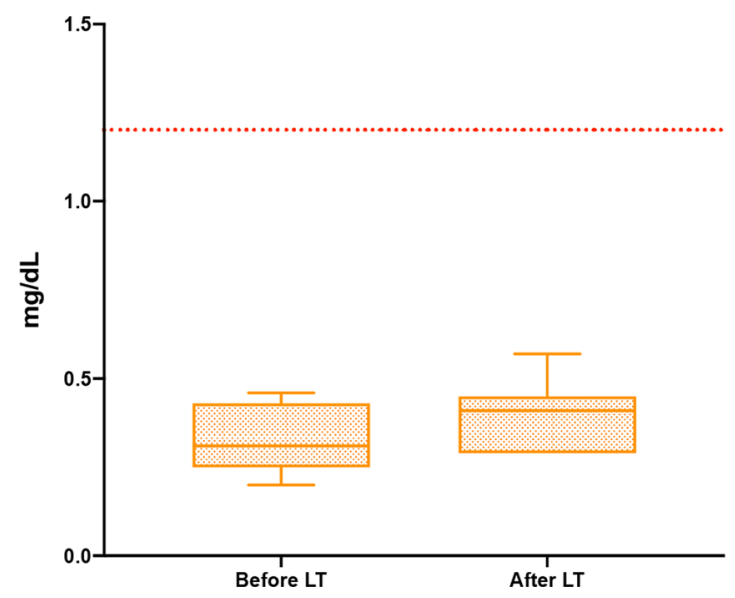

Fig. 3 Serum creatinine and estimated glomerular filtration rate (eGFR) before and after liver transplantation

eGFR

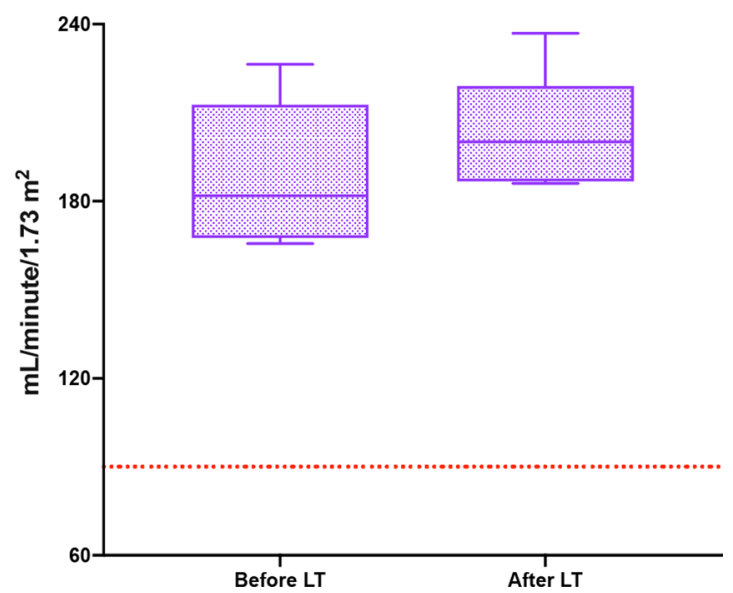

post-transplant follow-up. Pre-LT echocardiography was normal in all patients except for Patient 5. She displayed a mild left ventricular dilation and hypokinesia, and the echocardiogram results completely normalized with improved cardiac dilatation and left ventricular function 13.8 months following her transplant. Post-transplant routine echocardiography of other patients was normal.

The neurodevelopmental evaluation using DQ indicated that all patients suffered delayed neurological development prior to LT. Notably, the postoperative DQ levels improved or remained stable in all recipients (Table 2). Despite the improvements in neurodevelopmental delay, all our subjects still did not reach the values appropriate for their age.

\section{Postoperative body growth}

Body growth parameters, namely height and weight $\mathrm{Z}$ scores, were collected before and after LT. There were trends indicating an increase in height $\mathrm{Z}$ scores (preLT, $-0.76 \pm 1.48 ; \quad$ post-LT, $-0.51 \pm 1.23 ; \quad P=0.39)$ and a significant difference between preoperative and postoperative values were observed in weight $Z$ scores (preLT, $-0.96 \pm 1.19$; post-LT, $-0.21 \pm 1.26 ; P=0.04$ ) (Fig. 4).

\section{The hepatic expressions of PCCA and PCCB in the heterozygous living donor}

The hepatic expressions of PCCA and PCCB in one of the heterozygous parental living donors (Donor 7) were assessed at the protein level and compared to the liver specimens obtained from the PA patient (Patient 7) and a healthy donor. Western blotting using antibodies directed against PCCA and PCCB proteins demonstrated very low levels of PCCA and PCCB in the PA patient's liver. However, the hepatic PCCA expression in the heterozygous donor was higher than that of the normal control $(P<0.01)$, while the levels of PCCB were comparable $(P>0.05)$ (Fig. 5).

\section{Discussion}

To our knowledge, our series of 7 pediatric PA patients treated with LDLT is the largest to date from a single center. Of the 7 parental living donors, 6 were genetically proven obligate heterozygous carriers. In our report, all 7 recipients achieved satisfactory clinical outcomes with $100 \%$ patient and graft survival. There was no occurrence of metabolic decompensation, disease-associated longterm extrahepatic sequelae, or severe LT-related complications during a median follow-up period of 23.9 months. Meanwhile, all living donors recovered well after surgery, without metabolic acidosis or procedure-related complications during the immediate and long-term postoperative periods. More importantly, for the first time, we found that at the protein level, the hepatic expressions of PCCA and PCCB in the heterozygous donor were comparable to the healthy donor.

PA is a rare inborn error of mitochondrial metabolism with life-threatening consequences and multiorgan pathology. Despite following a strict protein restriction and pharmacological intervention, PA patients still suffer frequent metabolic decompensations and subsequent devastating long-term complications $[6,7,18]$. As a kind of enzyme replacement therapy, LT has been performed in 67 PA patients with 75 liver grafts to date [10, 11, 13$15,19-34]$. The overall patient and graft survivals were $80.6 \%$ and $72.0 \%$, respectively (Table 3 ). In the present study, despite a relatively short follow-up of 23.9 months 

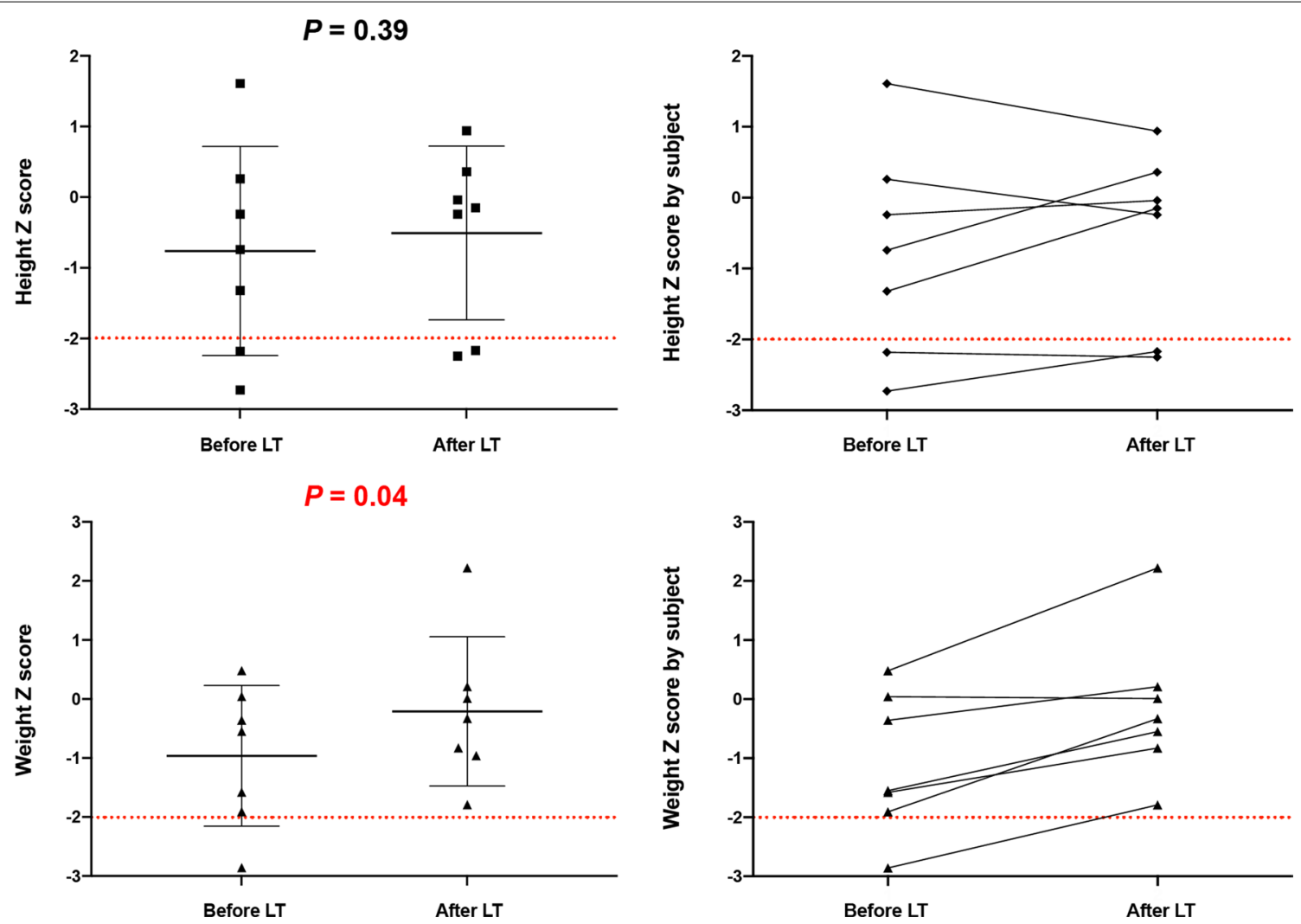

Fig. 4 Physical growth parameters (height and weight Z scores) before and after liver transplantation

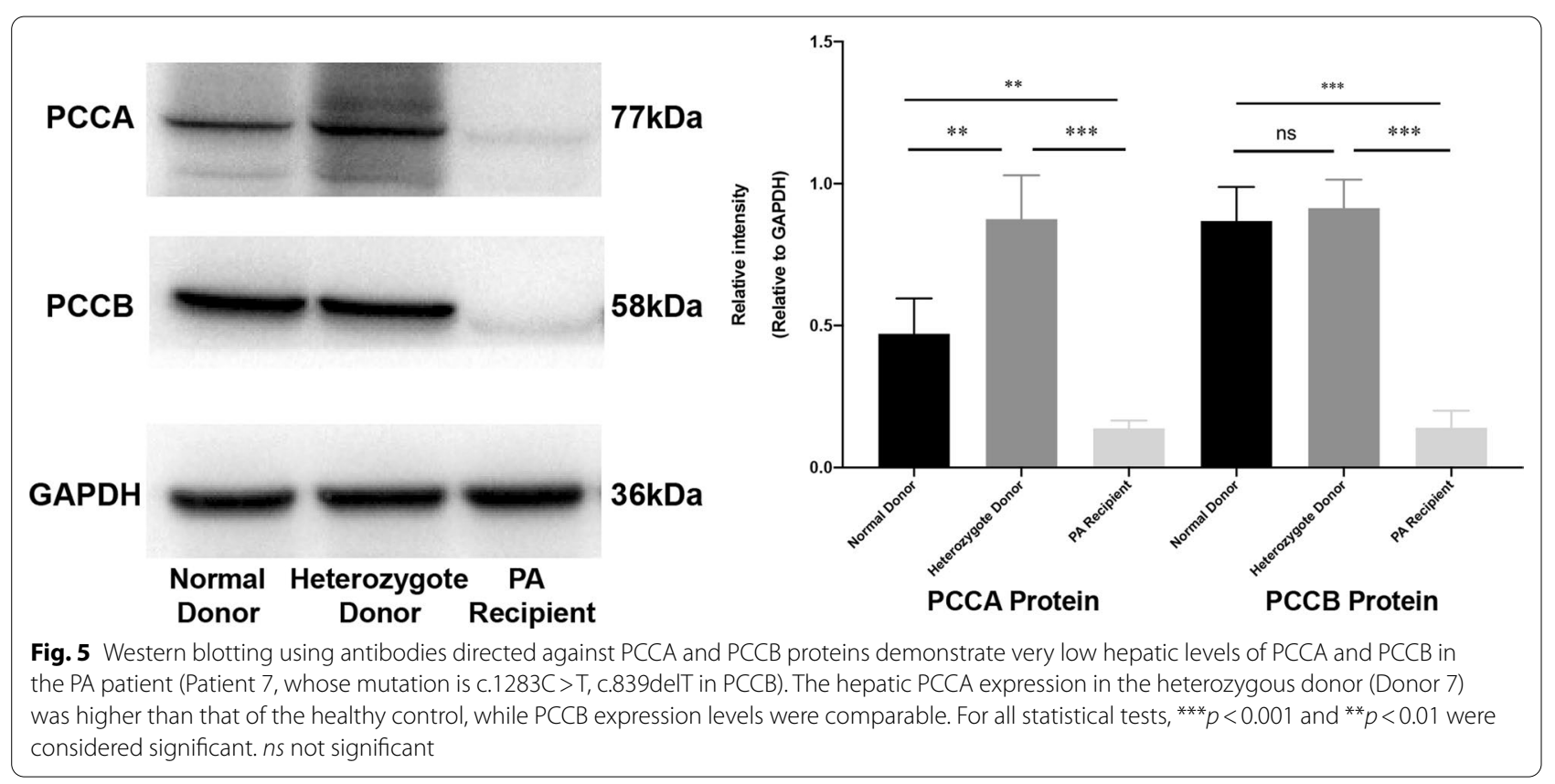




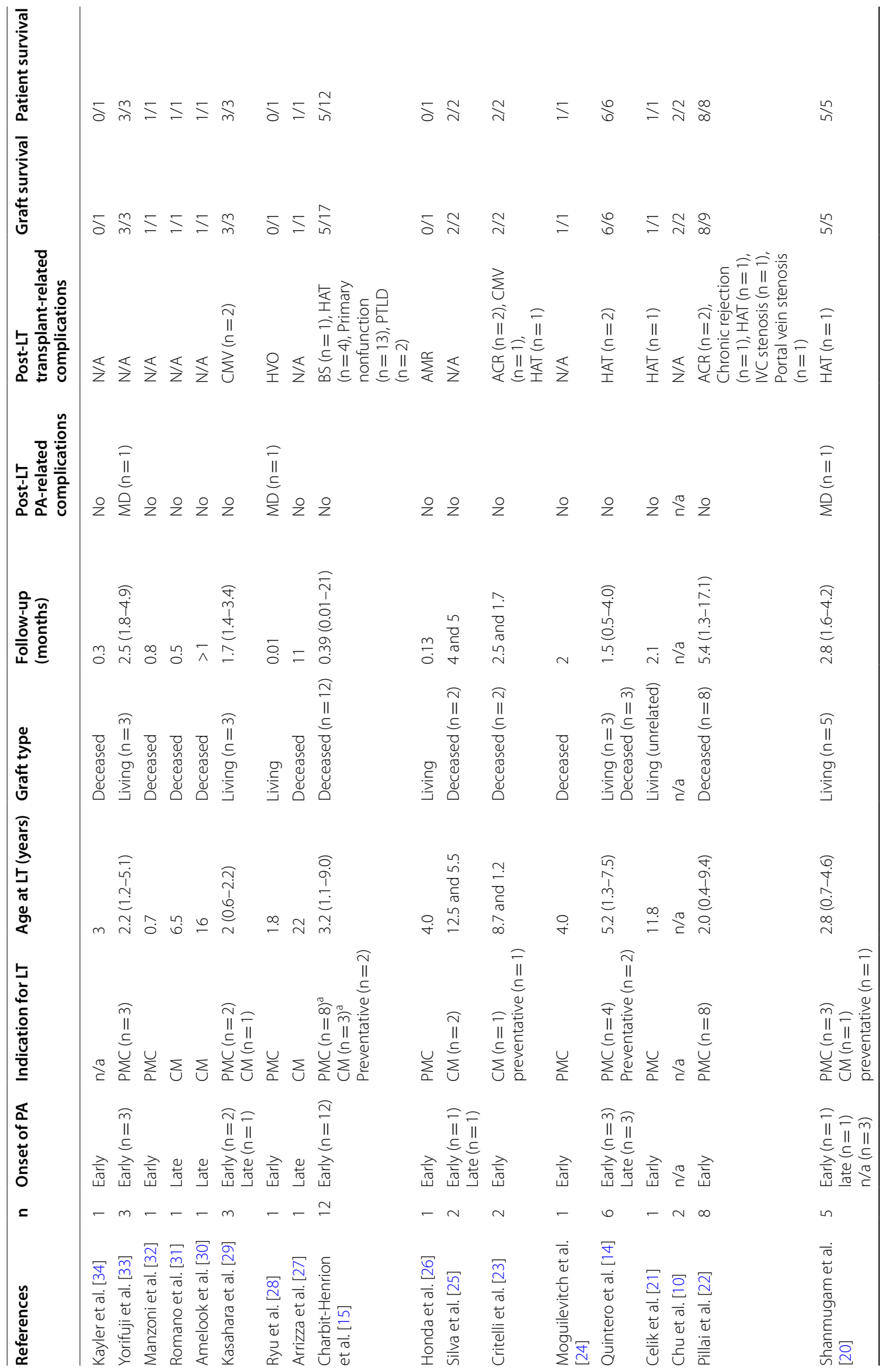




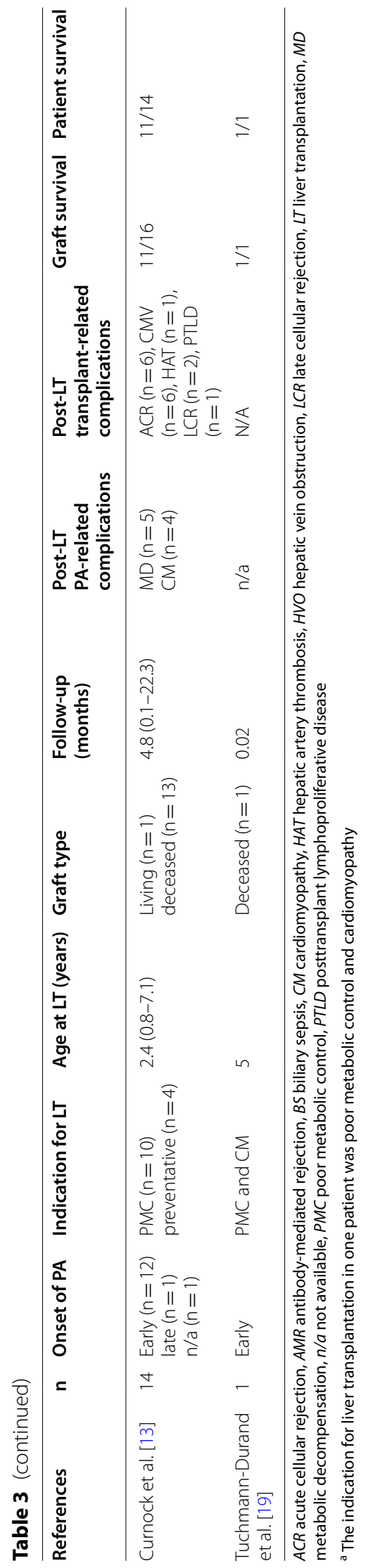


(13.9-40.2 months), our patients' survival outcomes were excellent, with $100 \%$ patient and graft survival rates, which were superior to previous data. These improvements may be attributed to the tremendous advances in surgical technology, medical management, and immunosuppressive agents since the 1990s. Hepatic artery thrombosis (HAT), one of the most life-threatening LT-related complications, is more frequent in pediatric LT, with an estimated rate of 4-8\% [35]. However, the overall rate of HAT in historical PA cases was significantly high at $17.5 \%$ [11]. Therefore, it was once believed that patients with PA are more prone to develop HAT after LT [14, 15]. Contrary to previous results, none of our patients developed HAT post-transplant, and thus we could not confirm a propensity for developing HAT in the liver transplant recipients with PA. Regarding other LT-related complications, neither our study nor previous studies have found that patients with PA were at higher risk. Therefore, concerns about transplant-related complications should not be an obstacle for PA patients to choose LT as a treatment option.

Frequent metabolic decompensations are the most common complication of PA patients, leading to frequent hospitalizations and impaired quality of life, and even being life-threatening. Thus, poor metabolic control has become the main indication for LT. Previous studies have demonstrated that LT reduces the risk of metabolic decompensation and improves the quality of life of PA patients $[13,14,22,29]$. In our study, 6 of the 7 recipients received LT due to frequent metabolic decompensations. Despite not all returning to normal levels, in the case of liberalized protein intake, propionate metabolites in our patients' blood and urine more or less decreased post-transplant. More importantly, no patients suffered further episodes of metabolic decompensation after LT. Therefore, it should be recognized that LT does bring metabolic stability to the already medically fragile PA patients, thereby largely protecting against metabolic decompensation and the need for frequent hospitalizations, which in itself leads to improved quality of life.

Cardiomyopathy, either dilated or hypertrophic, is another common and potentially lethal complication in PA, with an estimated incidence of 9-23\% $[4,36]$. It also contributes to one of the major causes of mortality in patients with PA [37]. The cardioprotective potential of LT for individuals with PA has been proved that reversal of cardiomyopathy is achieved in all previously reported 11 patients with pre-existing PA-associated cardiomyopathy $[15,20,23,25,27,29-31]$. In line with previous results, one of our patients with pre-existing mild dilated cardiomyopathy displayed a complete recovery of cardiac function after LT. Taken together, LT can be a viable therapeutic option for PA-related cardiomyopathy, and thus severe drug-resistant cardiomyopathy can remain as an indication for LT in patients with PA. Moreover, LT should not be contraindicated in PA patients with severely impaired cardiac function. Devices to stabilize the hemodynamic conditions, such as left ventricular assist device or extracorporeal membrane oxygenation, can be used as a bridge to LT $[29,30]$.

Neurodevelopmental sequelae are of particular concern in PA patients and the most crucial factor affecting the long-term quality of life. The poor neurocognitive and psychosocial development resulting from metabolic derangement was reported in $43-75 \%$ of PA patients [6, 38]. Previous studies suggested that almost all patients presented with neurodevelopmental delay prior to LT made some developmental progress after LT [13, 14, 22]. Our pretransplant neurodevelopmental assessment indicated that all individuals exhibited neurodevelopmental delay. However, LDLT has stabilized each patient's neurological impairment and even improved neurodevelopmental delay to some extent. Nevertheless, neurodevelopmental delay still exists in all surviving patients, and whether sustained neurological improvement could be expected requires more investigations in a longer follow-up.

Sufficient daily intake of essential and functional amino acids is necessary for normal body growth in children [39]. However, PA patients must follow a strict lifelong protein-restricted diet, resulting in severe amino acid deficiencies, so they are prone to develop body growth retardation [40]. A post-transplant liberalized protein diet means sufficient intake of essential and functional amino acids, which is crucial to correct chronic malnutrition and stimulate body growth in liver transplant recipients with PA. In turn, optimal growth leads to higher protein tolerance, which possibly helps further to reduce the risk of metabolic decompensation after transplantation [40]. In our series, in the case of no formal protein restriction, post-transplant mean height and weight $\mathrm{Z}$ scores were both improved compared with the pretransplant levels. However, whether the present patients' physical growth would catch up to the standard growth curve warrants further long-term follow-up.

Given the shortage of available donor organs and low priority in the waiting list, LDLT has been a feasible choice for inborn errors of metabolism, in which the donor is almost always a blood relative of the patient, and parental donors are preferable $[41,42]$. Since most monogenetic diseases are inherited in an autosomal recessive fashion, the parent who serves as a living donor is almost always an obligate heterozygous carrier. However, whether using a partial allograft from a heterozygous living donor contributes to sufficient metabolic correction in a homozygous recipient remains a concern. There is a possibility that the implanted 
partial liver graft may have low PCC activity, leading to persistent insufficient enzyme activity in the recipient, which will compromise the therapeutic value of LDLT in PA. There is also the possibility that the residual liver's PCC activity in the heterozygous donor is insufficient, which may put the donor in danger of disease-related symptoms and complications. Curnock et al. [13] hold the view that it should be preferential to use an unrelated LT donor in patients with PA. In contrast, previous studies suggested that no mortality or morbidity associated with the use of heterozygous carrier donors was observed in donors or recipients $[16,29,33,41]$. Our study is believed to be the largest reported series of PA patients receiving partial liver grafts from genetically proven obligate heterozygous carriers. We demonstrated satisfactory clinical outcomes in all 6 recipients, with no negative impact on both donors' and recipients' clinical courses. More importantly, for the first time, our study demonstrated that the hepatic expressions of PCCA and PCCB at the protein level in one of the heterozygous parental living donors were equal to those of the healthy donor. These results clearly indicate that LDLT using obligate heterozygous carriers as donors is a viable therapeutic option for PA. An early LT (ideally within the first year of life) has been considered for younger children with non-severe PA [13-15], and scheduled LT, according to the state of the disease, can almost only be performed in the setting of LDLT. Considering the promising therapeutic value of LDLT in the treatment of PA, LDLT using obligate heterozygous carriers as donors could be considered for selected PA patients, especially in countries with a limited deceased donor pool.

Although LT can theoretically provide a lifelong supply of PCC activity within the allograft, successful transplantation does not result in a cure in individuals with PA due to the ubiquitous enzyme deficiency throughout the body. Other extrahepatic tissues with remaining PCC deficiency, such as skeletal muscle, brain, heart, and kidneys, persistently produce pathognomonic propionate metabolites after LT [43]. These circulating toxic metabolites in turn cause damage to the central nervous system, heart, kidneys, and other organs throughout the body. Therefore, continued levocarnitine supplementation in the posttransplant period is advocated for all patients to enhance the excretion of propionate metabolites. Nevertheless, some liver transplant recipients with PA have been reported to develop metabolic decompensation, cardiomyopathy, and kidney dysfunction during long-term follow-up [13-15, 20, 28, 33, 44]. Although our patients did not experience the above-mentioned PA-related complications, it is recognized that LT can only achieve partial clinical improvements of this devastating disease and delay the progression of the disease or complications but cannot completely change the natural history of PA. The possibility of post-transplant non-remission or even chronic progression of the disease, especially the occurrence of long-term diseaserelated complications, should always be kept in mind by transplant surgeons. And patients' parents or guardians should be fully informed of these potential risks before LT. Lifelong regular follow-up, including metabolic, cardiac, renal, and neurological monitoring and evaluation, is necessary for all individuals with PA post-transplant. An experienced interdisciplinary team consisting of metabolism physicians, pediatric hepatologists, pediatric neurologists, pediatric cardiologists, pediatric nephrologists, pediatric transplantation team, metabolic dieticians, and neurorehabilitation physicians is also essential for the long-term management of patients with PA.

This study's limitations included its single-center retrospective nature, small sample size, and relatively shortterm follow-up. Nevertheless, we found for the first time that the hepatic expressions of PCCA and PCCB in the heterozygous parental living donor were equal to those of the healthy donor, which provides a solid foundation for the clinical use of LDLT from obligate heterozygous donors in patients with PA.

\section{Conclusion}

In summary, our study provides evidence that the hepatic expressions of PCCA and PCCB from the heterozygous donor are comparable to those of the healthy control at the protein level. LDLT using partial liver grafts from asymptomatic obligate heterozygous carrier donors is a viable therapeutic option for selected patients with PA, with no negative impact on both donors' and recipients' postoperative courses.

\section{Abbreviations}

ACR: Acute cellular rejection; AMR: Antibody-mediated rejection; BS: Biliary sepsis; CM: Cardiomyopathy; CMV: Cytomegalovirus; DQ: Developmental quotient; EBV: Epstein-Barr virus; eGFR: Estimated glomerular filtration rate; HAT: Hepatic artery thrombosis; HVO: Hepatic vein obstruction; ICU: Intensive care unit; GDS-C: Griffiths Development Scales-Chinese; LT: Liver transplantation; LDLT: Living donor liver transplantation; MDs: Metabolic decompensations; n/a: Not available; PA: Propionic academia; PCC: Propionyl-CoA carboxylase; PMC: Poor metabolic control; PTLD: Posttransplant lymphoproliferative disease; WISC-IV: Wechsler Intelligence Scale for Children-IV.

\section{Acknowledgements}

We acknowledge all patients and their families for participating in this study.

\section{Authors' contributions}

Z-JZ and L-YS conceptualized and designed the study and contributed to data interpretation. Z-GZ and G-PZ participated in designing the study, designed the data collection instruments, collected data, carried out the initial analyses, and drafted the initial manuscript. LW, WQ, YL, Y-LT, and JW participated in designing the study and coordinated and supervised data collection. All authors critically reviewed and revised the manuscript, approved the final manuscript as submitted, and agreed to be accountable for all aspects of the work. 


\section{Funding}

This work was supported by grants from Capital's Funds for Health Improvement and Research (Grant/Award Number: 2020-1-2024) and the National Natural Science Foundation of China (Grant/Award Number: 81970562).

\section{Availability of data and materials}

All data generated or analyzed during this study are included in this published article.

\section{Declarations}

\section{Ethics approval and consent to participate}

Written informed consent was obtained from all participants, and our study was approved by the Ethical Committee of Beijing Friendship Hospital, Capital Medical University (No. 2020-P2-094-01).

\section{Consent for publication}

Not applicable.

\section{Competing interests}

All authors declare no conflict of interest to disclose.

\section{Author details}

'Liver Transplantation Center, National Clinical Research Center for Digestive Diseases, Beijing Friendship Hospital, Capital Medical University, No. 101 Lu Yuan Dong Road, Tong-Zhou District, Beijing 101100, China. Department of Critical Liver Diseases, Liver Research Center, Beijing Friendship Hospital, Capital Medical University, Beijing 101100, China. ${ }^{3}$ Clinical Center for Pediatric Liver Transplantation, Capital Medical University, Beijing 101100, China.

Received: 5 July 2021 Accepted: 6 February 2022

Published online: 21 February 2022

\section{References}

1. Almasi T, Guey LT, Lukacs C, Csetneki K, Voko Z, Zelei T. Systematic literature review and meta-analysis on the epidemiology of propionic acidemia. Orphanet J Rare Dis. 2019;14(1):40.

2. Wongkittichote $P$, Ah Mew N, Chapman KA. Propionyl-CoA carboxylasea review. Mol Genet Metab. 2017;122(4):145-52.

3. Kolker S, Garcia-Cazorla A, Valayannopoulos V, Lund AM, Burlina AB, Sykut-Cegielska J, et al. The phenotypic spectrum of organic acidurias and urea cycle disorders. Part 1: the initial presentation. J Inherit Metab Dis. 2015:38(6):1041-57.

4. Haijes HA, Jans JJM, Tas SY, Verhoeven-Duif NM, van Hasselt PM. Pathophysiology of propionic and methylmalonic acidemias. Part 1: complications. J Inherit Metab Dis. 2019;42(5):730-44.

5. Jurecki E, Ueda K, Frazier D, Rohr F, Thompson A, Hussa C, et al. Nutrition management guideline for propionic acidemia: an evidence- and consensus-based approach. Mol Genet Metab. 2019;126(4):341-54.

6. Grünert SC, Müllerleile S, De Silva L, Barth M, Walter M, Walter K, et al. Propionic acidemia: clinical course and outcome in 55 pediatric and adolescent patients. Orphanet J Rare Dis. 2013:8:6.

7. Shchelochkov OA, Manoli I, Sloan JL, Ferry S, Pass A, Van Ryzin C, et al. Chronic kidney disease in propionic acidemia. Genet Med. 2019;21(12):2830-5

8. Forny P, Horster F, Ballhausen D, Chakrapani A, Chapman KA, Dionisi-Vici $C$, et al. Guidelines for the diagnosis and management of methylmalonic acidaemia and propionic acidaemia: first revision. J Inherit Metab Dis. 2021;44(3):566-92.

9. Jiang YZ, Shi Y, Shi Y, Gan LX, Kong YY, Zhu ZJ, et al. Methylmalonic and propionic acidemia among hospitalized pediatric patients: a nationwide report. Orphanet J Rare Dis. 2019;14(1):292.

10. ChuTH, Chien YH, Lin HY, Liao HC, Ho HJ, Lai CJ, et al. Methylmalonic acidemia/propionic academia - the biochemical presentation and comparing the outcome between liver transplantation versus non-liver transplantation groups. Orphanet J Rare Dis. 2019;14(1):73.
11. Zhou GP, Jiang YZ, Wu SS, Kong YY, Sun LY, Zhu ZJ. Liver transplantation for propionic acidemia: evidence from a systematic review and metaanalysis. Transplantation. 2021;105(10):2272-82.

12. Molema F, Martinelli D, Hörster F, Kölker S, Tangeraas T, de Koning B, et al. Liver and/or kidney transplantation in amino and organic acid-related inborn errors of metabolism: an overview on European data. J Inherit Metab Dis. 2021:44(3):593-605.

13. Curnock R, Heaton ND, Vilca-Melendez H, Dhawan A, Hadzic N, Vara R. Liver transplantation in children with propionic acidemia: medium-term outcomes. Liver Transpl. 2020;26(3):419-30.

14. Quintero J, Molera C, Juamperez J, Redecillas S, Meavilla S, Nunez R, et al. The role of liver transplantation in propionic acidemia. Liver Transpl. 2018;24(12):1736-45.

15. Charbit-Henrion F, Lacaille F, McKiernan P, Girard M, de Lonlay P, Valayannopoulos $V$, et al. Early and late complications after liver transplantation for propionic acidemia in children: a two centers study. Am J Transplant. 2015;15(3):786-91.

16. Nagao M, Tanaka T, Morii M, Wakai S, Horikawa R, Kasahara M. Improved neurologic prognosis for a patient with propionic acidemia who received early living donor liver transplantation. Mol Genet Metab. 2013;108(1):25-9.

17. Li PY, Fu NN, Li OY, Wang GF, Gao L, Zhang X. The Griffiths Development Scales-Chinese (GDS-C): a reliable and valid neurodevelopmental assessment tool in children with ASD aged 3-8 years old in Tianjin, China. Asian J Psychiatr. 2020;52:1144.

18. Riemersma M, Hazebroek MR, Helderman-van A, den Enden GS, Salomons SF, Brouwers $M$, et al. Propionic acidemia as a cause of adult-onset dilated cardiomyopathy. Eur J Hum Genet. 2017;25(11):1195-201.

19. Tuchmann-Durand C, Thevenet E, Moulin F, Lesage F, Bouchereau J, Oualha M, et al. Administration of gamma-hydroxybutyrate instead of beta-hydroxybutyrate to a liver transplant recipient suffering from propionic acidemia and cardiomyopathy: a case report on a medication prescribing error. JIMD Rep. 2020;51(1):25-9.

20. Shanmugam NP, Valamparampil JJ, Reddy MS, Al Said KJ, Al-Thihli K, Al-Hashmi N, et al. Auxiliary partial orthotopic liver transplantation for monogenic metabolic liver diseases: single-centre experience. JIMD Rep. 2019;45:29-36

21. Celik N, Squires JE, Soltys K, Vockley J, Shellmer DA, Chang W, et al. Domino liver transplantation for select metabolic disorders: expanding the living donor pool. JIMD Rep. 2019;48(1):83-9.

22. Pillai NR, Stroup BM, Poliner A, Rossetti L, Rawls B, Shayota BJ, et al. Liver transplantation in propionic and methylmalonic acidemia: a single center study with literature review. Mol Genet Metab. 2019;128(4):431-43.

23. Critelli K, McKiernan P, Vockley J, Mazariegos G, Squires RH, Soltys K, et al. Liver transplantation for propionic acidemia and methylmalonic acidemia: perioperative management and clinical outcomes. Liver Transpl. 2018:24(9):1260-70.

24. Moguilevitch M, Delphin E. Domino liver transplantation from a child with propionic acidemia to a child with idiopathic fulminant hepatic failure. Case Rep Transplant. 2018;2018:1897495.

25. Silva HM, Nassogne MC, Smets F, Stephenne X, Scheers I, Veyckemans F, et al. Liver transplantation for propionic acidemia. J Pediatr Gastroenterol Nutr. 2017:64(3):e73-6.

26. Honda M, Sakamoto S, Sakamoto R, Matsumoto S, Irie T, Uchida K, et al. Antibody-mediated rejection after $\mathrm{ABO}$-incompatible pediatric living donor liver transplantation for propionic acidemia: a case report. Pediatr Transplant. 2016;20(6):840-5.

27. Arrizza C, De Gottardi A, Foglia E, Baumgartner M, Gautschi M, Nuoffer JM Reversal of cardiomyopathy in propionic acidemia after liver transplantation: a 10-year follow-up. Transpl Int. 2015;28(12):1447-50.

28. Ryu J, Shin YH, Ko JS, Gwak MS, Kim GS. Intractable metabolic acidosis in a child with propionic acidemia undergoing liver transplantation-a case report. Korean J Anesthesiol. 2013;65(3):257-61.

29. Kasahara M, Sakamoto S, Kanazawa H, Karaki C, Kakiuchi T, Shigeta T, et al. Living-donor liver transplantation for propionic acidemia. Pediatr Transplant. 2012;16(3):230-4.

30. Ameloot K, Vlasselaers D, Dupont M, Meersseman W, Desmet L, Vanhaecke J, et al. Left ventricular assist device as bridge to liver transplantation in a patient with propionic acidemia and cardiogenic shock. J Pediatr. 2011;158(5):866-7. 
31. Romano S, Valayannopoulos V, Touati G, Jais JP, Rabier D, de Keyzer Y, et al. Cardiomyopathies in propionic aciduria are reversible after liver transplantation. J Pediatr. 2010;156(1):128-34.

32. Manzoni D, Spotti A, Carrara B, Gritti P, Sonzogni V. Anaesthesia for liver transplantation in two infants with an organic acidaemia. Pediatr Transplant. 2006;10(5):623-8.

33. Yorifuji T, Kawai M, Mamada M, Kurokawa K, Egawa H, Shigematsu Y, et al. Living-donor liver transplantation for propionic acidaemia. J Inherit Metab Dis. 2004:27(2):205-10.

34. Kayler LK, Merion RM, Lee S, Sung RS, Punch JD, Rudich SM, et al. Long-term survival after liver transplantation in children with metabolic disorders. Pediatr Transplant. 2002;6(4):295-300.

35. Kohli R, Cortes M, Heaton ND, Dhawan A. Liver transplantation in children: state of the art and future perspectives. Arch Dis Child. 2018;103(2):192-8.

36. Baumgartner MR, Hörster F, Dionisi-Vici C, Haliloglu G, Karall D, Chapman $\mathrm{KA}$, et al. Proposed guidelines for the diagnosis and management of methylmalonic and propionic acidemia. Orphanet J Rare Dis. 2014;9:130.

37. Kovacevic A, Garbade SF, Hoffmann GF, Gorenflo M, Kolker S, Staufner C. Cardiac phenotype in propionic academia-results of an observational monocentric study. Mol Genet Metab. 2020;130(1):41-8.

38. Fujisawa D, Nakamura K, Mitsubuchi H, Ohura T, Shigematsu Y, Yorifuji T, et al. Clinical features and management of organic acidemias in Japan. J Hum Genet. 2013;58(12):769-74.

39. Lui JC, Garrison P, Baron J. Regulation of body growth. Curr Opin Pediatr. 2015;27(4):502-10.

40. Molema F, Gleich F, Burgard P, van der Ploeg AT, Summar ML, Chapman $K A$, et al. Decreased plasma l-arginine levels in organic acidurias (MMA and PA) and decreased plasma branched-chain amino acid levels in urea cycle disorders as a potential cause of growth retardation: options for treatment. Mol Genet Metab. 2019;126(4):397-405.

41. Morioka D, Takada Y, Kasahara M, Ito T, Uryuhara K, Ogawa K, et al. Living donor liver transplantation for noncirrhotic inheritable metabolic liver diseases: impact of the use of heterozygous donors. Transplantation. 2005;80(5):623-8

42. Jiang YZ, Sun LY, Zhu ZJ, Wei L, Qu W, Zeng ZG, et al. Perioperative characteristics and management of liver transplantation for isolated methylmalonic acidemia-the largest experience in China. Hepatobiliary Surg Nutr. 2019;8(5):470-9.

43. Chapman KA, Summar ML, Enns GM. Propionic acidemia: to liver transplant or not to liver transplant? Pediatr Transplant. 2012;16(3):209-10.

44. Sivananthan S, Hadžić N, Dhawan A, Heaton ND, Vara R. Fatal metabolic stroke in a child with propionic acidemia 11 years post liver transplant. Am J Transplant. 2021;21(4):1637-40.

\section{Publisher's Note}

Springer Nature remains neutral with regard to jurisdictional claims in published maps and institutional affiliations.

Ready to submit your research? Choose BMC and benefit from:

- fast, convenient online submission

- thorough peer review by experienced researchers in your field

- rapid publication on acceptance

- support for research data, including large and complex data types

- gold Open Access which fosters wider collaboration and increased citations

- maximum visibility for your research: over $100 \mathrm{M}$ website views per year

At BMC, research is always in progress.

Learn more biomedcentral.com/submissions 